\author{
Using Transfer of Stimulus Control Technology to Promote \\ Generalization and Spontaneity of Language \\ Trina D. Spencer, $\mathrm{PhD}$ \\ Northern Arizona University \\ Thomas S. Higbee, PhD \\ Utah State University
}

Author Contact Info:

Trina D. Spencer

Northern Arizona University

Institute for Human Development

PO Box 5630

Flagstaff, AZ 86011-5630

Trina.Spencer@nau.edu

Acknowledgements: The first author would like to express gratitude to the research assistants, students, and administration of the Autism Support Services: Education, Research and Training (ASSERT) program for their commitment to excellence through research. 


\begin{abstract}
Children with autism often use newly acquired language in restricted contexts and with limited variability. Instructional tactics that embed generalization technology have shown promise for increasing spontaneity, response variation, and the generalized use of language across settings, people, and materials. This case example explores the integration of textual scripts and explicit engineering of transfer of stimulus control procedures to facilitate functional conversation skills of a young girl with autism. The generalized use of prepositions, coordinating conjunctions, and subordinating conjunctions was assessed within the context of natural conversation with teachers, parents, and peers. The intentional programming of training contexts has the potential to impact language spontaneity, generalization, and variability without relying on script fading conventions.
\end{abstract}

Keywords: script training, generalization, language, autism, conversation 
Using Transfer of Stimulus Control Technology to Promote Generalization and Spontaneity of Language: A Case Example

Significant language deficits and impaired social interactions are characteristic of children with autism (American Psychiatric Association, 2000). Many children with autism display limited spontaneity and generalization of newly acquired language skills (Carr \& Kologinsky, 1983; Charlop, Schreibman, \& Thibodeau, 1985: Koegel, 2000) and engage socially only in response to questions or prompts from adults and in tightly controlled training environments (Chiang \& Carter, 2008; Sigafoos \& Reichle, 1993). For example, a child with autism may only talk about his or her current activity when directly asked about it and after several training sessions, in contrast to typical children, who seek out friends, initiate conversations with questions or comments, and use language appropriate for the immediate context.

For language skills to be functional their use needs to become spontaneous, to occur in a variety of non-training situations, and to extend beyond directly taught statements. Spontaneity is typically discussed with respect to specific programmed or unprogrammed antecedent stimuli that prompt targeted language behaviors. Rather than identifying something as spontaneous or not spontaneous, though, spontaneity can be conceived as the extent to which the discriminative stimuli that evoke language form and use occur naturally in conversation contexts (Carter \& Hotchkis, 2002; Sigafoos \& Reichle, 1993). The degree of intrusiveness of the controlling stimuli or the saliency of contextual events is also relevant. The less obvious and intrusive the discriminative stimuli, the more spontaneous the response may be considered (Halle, 1987). For example, language evoked by a conversation partner and the presence of toys is more spontaneous than language evoked by a teacher's vocal prompt, because explicit prompting from 
adults is intrusive and does not occur in natural conversation. Training for spontaneity involves the careful arrangement of antecedent stimuli to promote the transfer of stimulus control from programmed stimuli such as adult prompting to unprogrammed stimuli such as the presence of toys and conversation partners (e.g., Argott, Townsend, Sturey, \& Poulson, 2008; Brown, Krantz, McClannahan, \& Poulson, 2008; Krantz \& McClannahan, 1993). This transfer of stimulus control occurs through a process of pairing the programmed stimuli (e.g., prompts) with antecedent stimuli that are typically present in the natural, non-training environments. Gradual fading of the programmed stimuli can facilitate the transfer (Cooper, Heron, \& Heward, 2007; Stokes \& Baer, 1977).

In much of the language research conducted with children with autism, stimulus/situation generalization is assessed. This type of generalization involves the transfer of stimulus control of acquired skills from a set of stimuli present in the training environment (e.g., conversation partners and setting of interaction) to similar stimuli that were not present during training (Cooper et al., 2007; Stokes \& Baer, 1977). For instance, a child who was taught to say, "Look" to a teacher sitting at a desk might later emit the response "Look" in the presence of a peer on the playground.

Another way to conceptualize generalization is by determining the degree of flexibility in the use of newly acquired language. Response generalization occurs when one response is taught and then, following training, the child emits one or more different but functionally equivalent responses (Cooper et al., 2007). For example, a child is taught, "Look what I can do" and later, without any direct training on response forms, he or she uses a different but functionally equivalent response such as, "Watch what I am doing." In response generalization, the antecedent stimuli present in the conversation context have control over a set of functionally 
equivalent responses or a response class, which might be a looser type of stimulus control than if they controlled only one specific response form.

Many children with autism require systematic and carefully engineered training programs before they experience significant generalized behavior change (Koegel, 2000; McConnell, 2002). Often, training begins with the acquisition of new verbal responses and, following proficient use of those responses under controlled training conditions, the focus of training changes to pragmatics - spontaneity and generalization. In initial training, reinforcement of specific forms of language exclusively in the presence of programmed antecedent stimuli may hinder the transfer of stimulus control to non-training antecedent stimuli and settings, and restrict response variations. Several researchers have noted that precise stimulus control desired during initial training with children with autism may interfere with the spontaneous and generalized use of the newly acquired verbal responses (Carr, 1982; Carr \& Kologinsky, 1983; Sigafoos \& Reichle, 1993). Given that children with autism may need systematic training to use language functionally and that narrow stimulus control can impair spontaneity and generalization, procedures that balance effective acquisition with the strategic transfer of stimulus control are needed.

One procedure that was designed to enhance the spontaneous and generalized use of language of children with autism is script training. To reduce the need for intrusive vocal prompting from adults, auditory or written phrases, managed by the child, are used to prompt targeted language utterances. Children are first taught to use scripts. This may involve pointing to and reading textual scripts or inserting Language Master Cards through a Language Master device and repeating audio scripts. Following initial script training procedures, scripts are typically faded by gradually eliminating parts of the scripts (McClannahan \& Krantz, 2005). 
Several researchers have investigated the effect of scripts and script fading on language skills of children with autism with varying degrees of success. In an early script intervention study, Krantz \& McClannahan (1993) arranged ten written statements or questions for the participants to read during leisure activities with a peer. Once participants were proficient responders, textual scripts were faded from end to beginning in five steps. During training and script fading, participants made conversational statements cued by the scripts or the remaining element(s) of the scripts. All scripts were enclosed with a set of quotation marks ("). They conducted a series of stimulus/situation generalization sessions in a different room, with a different teacher, at different times of day, and with different materials. With respects to response generalization, they measured unscripted initiations, which were defined as "verbal productions that differed from the script by more than conjunctions, articles, prepositions, pronouns, or changes in verb tense," (p. 124). Researchers programmed several features into the procedures to facilitate response variation. For example, scripts were developed to reflect recent or upcoming events and familiar school objects and three different versions of scripts were created by randomly assigning the position of scripts before presenting them. The results of this study were favorable as unscripted responses increased substantially and stimulus/situation generalization was achieved; however, when the scripts were faded completely, participants were unable to initiate conversation; the beginning quotation mark was necessary to evoke conversation. It is interesting to consider that, while quotation marks are not necessarily natural antecedent stimuli that typically control conversation, they are certainly less salient than the entire text script used in training. Moreover, the quotation marks, a non-specific discriminative stimulus, cued speaking in general rather than specific statements. Given that the discriminative stimulus (i.e., 
the quotation marks) was useable for a variety of potential scripts appropriate for the conversational context, the outcome is significant.

In a recent study, Brown, Krantz, McClannahan, and Poulson (2008) supplemented script training and script fading procedures with additional strategies to enhance the transfer of stimulus control of verbal responses to naturally-occurring stimuli. They taught participants nine conversation statements that corresponded to nine items commonly found in convenience stores, sporting good stores, and video stores. Textual scripts were attached to these items during mock store training and once participants responded proficiently using the scripts, scripts were faded from end to beginning in seven steps. Pre- and post-tests were conducted in community stores and results indicated participants emitted substantially more unprompted and unscripted interactions following script training and fading. Several features of their procedures facilitated natural environment stimulus control of conversational interactions. First, a subset of nine generalization stimuli was present during training; thus, multiple common stimuli were present during training and generalization settings. Second, the location of attached textual scripts on training stimuli was moved after each fading step. Third, manual prompting for participants to point to the script was provided only after 30 seconds of no talking and the conversation partners never began the interactions. Fourth, adult conversation partners responded to participants' attempts to interact with natural conversation responses. Social conversation responses were natural consequences of participants' initiations.

\section{Pilot Research and Background}

Approximately one year before the conduct of the current study, we attempted to investigate a set of procedures derived from the script training plus fading literature (e.g., Brown et al., 2008; Krantz \& McClannahan, 1993). Participants included three children (5-7 years old) 
with high functioning autism. Five audio scripts were taught and entered script fading sequentially to increase the spontaneity, generalization, and variability of conversational language. Training took place in the context of play (e.g., play-do, painting, games) with three rotating adult conversation partners. Two participants were given small edible treats to reinforce repetition of scripts and conversation partners were trained to be neutral throughout the sessions. Because performance criteria for fading scripts were common in the literature (e.g., MacDuff, Ledo, McClannahan, \& Krantz, 2007; Reagon \& Higbee, 2009; Stevenson, Krantz, \& McClannahan, 2000), we also followed a performance criterion for advancing to the next step of script fading, which was two consecutive sessions in which participants accurately and independently produced the scripts.

Results indicated that children's conversation increased when the audio recorder and audio scripts were present, however, they were never able to initiate conversation when the scripts were not available. In other words, the fading was unsuccessful. There are several observations worth mentioning as they relate to the design of the current procedures. During the script fading phases when words were missing from the script, participants often abandoned the current script and repeated a previously learned script instead. This was likely due to the sequential introduction of the scripts leading to greater exposure to earlier scripts (and reinforcement). One participant repeated only the portion of the script that was available during script fading, never completing the portion that had been removed and the others never altered what they said in response to the partial script. Apparently, they had memorized the scripts and never deviated from them (no response generalization was observed). We hypothesized this was because we inadvertently reinforced their behavior of following the script exactly by requiring the performance criterion for advancing to the next script fading step and through the number of 
sessions spent at each step. One child was more talkative with some activities than others. Based on our observations, we concluded that he preferred the one activity and disliked the others; thus only motivated to talk about the one. Lastly, some aspect of the audio scripts and/or the play context prevented successful transfer of stimulus control from the intrusive training stimuli (i.e., scripts) to the less intrusive, but programmed stimuli (i.e., toys and conversational partners). We speculated that it was because one stimulus was auditory and the other was visual; thus impeding in the pairability of the stimuli.

As a result of our pilot research experience, we became skeptical about the necessity of including the script fading phase. In our situation, script fading was very difficult to manage and it seemed to do the exact opposite of what we intended. While previous researchers supplemented script fading with various instructional arrangements to enhance spontaneity and generalization, the effort and duration of script fading was still significant (5-7 steps over 16-52 sessions). A different set of researchers adapted the script training intervention so that stimulus fading was unnecessary. Woods and Poulson (2006) employed a script training procedure without script fading to teach children with autism to initiate interactions with typical peers during art and play activities. Participants were prompted manually to use a binder filled with several textual scripts to cue initiations to peers. Although they were never faded during the training condition, scripts were not present during probe "lunch bunch" sessions. A variation of fading was accomplished by periodically exposing children to a similar context but without the scripts. When scripts were introduced, participants' scripted initiations increased significantly. During setting generalization probe sessions, initiations increased over baseline conditions, although they did not reach the level of initiations when scripts were present. Even though only moderate improvements in the generalization setting were noted, this outcome is important 
because it was accomplished in just a few training sessions (3-13) and without the difficult to manage script fading phase. Spontaneous and generalized language produced in fewer sessions and with less instructor effort has significant practical implications.

Although in our pilot research, participants' language spontaneity, generalization, and variability did not improve, the lessons learned were extremely helpful in designing a more successful approach. Drawing from this pilot work, known generalization technology (Stokes \& Baer, 1977), and previous script training research (Brown et al., 2008; Krantz \& McClannahan, 1993; Woods \& Poulson, 2006), we developed a number of hypotheses that led to the intentional integration of multiple strategies to enhance the transfer of stimulus control without requiring script fading: (1) introducing several scripts at the same time should prevent children from reverting to previously learned scripts and reduce the possibility that children can memorize each one exactly; (2) eliminating a performance criterion should reduce the inadvertent reinforcement of invariable responses; (3) including natural reinforcers (e.g., social attention instead of edibles) and highly preferred activities during training should eliminate motivation as a confound; and finally, (4) increasing the saliency of paired stimuli (making both visual) should improve the transfer of stimulus control potential between the intrusive programmed stimuli (i.e., scripts) and the natural stimuli (i.e., toys and conversation partner).

These hypotheses led to the current procedures and purpose, which was to explore the effect of supplementing script training with a number of strategies to promote generalization on the spontaneous and generalized use of complex language targets of a child with autism. Specifically we were interested in knowing if combining script training and generalization strategies during the acquisition phases enhance the transfer of stimulus control and if stimulus control could be transferred from scripts to more naturally occurring stimuli in the absence of 
formal script fading. We carefully arranged our teaching procedures to include the following generalization-enhancing tactics: a) natural contingencies of reinforcement, b) training multiple exemplars, c) training loosely, and d) programming common stimuli (Stokes \& Baer, 1977).

\section{Method}

\section{Participant and Setting}

Fran, a 5-year-old girl with high functioning autism, served as the participant in this case study. In general, Fran exhibited average to above average cognitive skills, but displayed significant behavior problems such as tantrums and routine inflexibility. Fran had functionally adequate language skills (i.e., she communicated her basic wants and needs) and spontaneously produced short, simple sentences. However, she often omitted smaller words like "to" and "am." For instance, taken from a transcribed language sample prior to the study, Fran said, "I making rainbow" and "I want be this one." While Fran was able to communicate with understanding adults, such as her parents and teachers, peers were not especially responsive to Fran's attempts to communicate. Despite her interest in being with typical peers, Fran did not consistently maintain eye contact with them or initiate conversation on topics of interest to her peers. Most of Fran's conversations occurred with adults and were initiated by adults.

Fran attended a university-based preschool program with seven other preschool-aged children. The program serves as a training center for undergraduate and graduate students who provide one-on-one intensive behavioral intervention to young children with autism. Of the eight children with autism in the program at the time, Fran was the only one who was interested in peers and could communicate sufficiently. Because she lacked a community of peers in this program, Fran was escorted twice a week to a campus daycare program during "free play" where she was encouraged to interact socially with typical peers. To prepare her for kindergarten in a 
less restrictive setting, Fran was an ideal candidate for a social communication intervention with an explicit focus on the generalized use of language.

Fran's instructors were undergraduate students in psychology or communication sciences. Instructors received extensive training in Applied Behavior Analysis and were closely supervised by doctoral candidates and the program's director. Fran worked with each instructor once or twice a week for four hours and research activities were integrated into her instructional time every day during the summer before she entered kindergarten.

For research purposes, we taught Fran conversational language in the context of art. Painting and coloring were some of Fran's favorite activities and sitting at a table with a conversation partner provided an appropriate level of structure for training. In kindergarten, Fran would have frequent opportunity to sit near peers during art class and kindergarten children typically talk about ongoing projects with peers. Training in this context prepared Fran for future social interactions in kindergarten.

The majority of sessions took place in the university-based preschool at a small table with her instructors. However, setting generalization probes were conducted in Fran's home at the dining room table and, at follow-up, in her mainstream kindergarten classroom at a large table during art class.

\section{Dependent Variable and Design}

The dependent variable was selected based on Fran's social language needs. We employed a language sample analysis to identify developmentally appropriate language features absent in Fran's conversations. Fran's language productions were recorded using a digital voice recorder during conversation with her instructor (constituted first three baseline assessment sessions). Following the recording of three 3-minute language samples, Fran's productions 
during conversation were transcribed and carefully reviewed for language features that she did not use (Robertson, 2007). After analyzing transcripts of Fran's first three baseline assessment sessions, we identified three language features and specific words to target in this study: a) prepositions on, in, and with, b) coordinating conjunctions and and but, and c) subordinating conjunctions when and because. The number of language targets Fran emitted during daily 3minute assessment sessions was the primary dependent measure.

A multiple baseline experimental design across three language features was employed for this study. Fran's conversation productions were assessed daily during baseline and intervention conditions, which lasted approximately 9 weeks. Every day, data for all three language targets were extracted from a single transcript of the assessment conversation. After four baseline sessions, in which Fran did not use the selected targets, we initiated training of prepositions scripts. The second language feature trained was coordinating conjunctions. Once Fran consistently used the previous two targets, we introduced subordinating conjunctions. All the daily assessment sessions took place in her preschool program with training materials and with her instructors serving as the conversation partner.

To capture information regarding setting/stimulus generalization, we conducted a variety of generalization probes, which differed from the daily assessment sessions by at least one dimension (e.g., conversation partner, setting, materials). We systematically probed Fran's use of prepositions, coordinating conjunctions, and subordinating conjunctions in non-training environments (home and kindergarten classroom), with non-training materials (stamps and stamp pad), and with non-training conversation partners (peers and mother) in baseline and following the 9 week intervention phase. A maintenance probe was conducted following a two-week summer break and after Fran's first two weeks in a general education kindergarten classroom 
(i.e., after a four-week period of no intervention). In lieu of having typical peers available in Fran's preschool program, the investigators brought their children in to serve as confederate peers when possible. The maintenance probe was also conducted with the first author's 5-yearold son because he was in Fran's mainstream kindergarten class.

\section{Independent Variable}

The independent variable included two major components - script training without fading and transfer of stimulus control procedures. The transfer of stimulus control procedures (or strategies to promote generalization) involved the systematic arrangement of multiple exemplars during training and the use of stimuli common to both the training and naturalistic assessment conditions. In addition, we intentionally trained the multiple exemplars loosely and incorporated natural contingencies of reinforcement in both training and assessment contexts (Stokes \& Baer, 1977). For this study, textual scripts prompted what to say, but the strategies to promote generalization were intentionally arranged to enhance the functional use of those statements.

\section{Procedures}

Assessment sessions occurred each morning (Monday through Friday) at approximately the same time. During the intervention phase, training sessions occurred about 15 minutes after the assessment sessions. The only difference between assessment and training sessions was that scripts and a prompter were not present during assessment sessions like they were in training sessions. Based on the preschool's established work schedule, one of three undergraduate instructors served as the conversation partner for the day and participated in both assessment (except for generalization probes with Fran's peers and mother) and training sessions. We arranged for transfer of stimulus control using stimuli common (i.e., art materials, familiar but rotating conversation partners, and a table) in both assessment and training sessions. The 
conversation partner sat across from Fran while painting with art supplies. We selected four types of art supplies, with which Fran had limited exposure. Intervention materials included: a) Color Wonder finger paints with Color Wonder paper, b) squeeze paints with art paper, and c) dot paints with art paper. Flower and heart stamps, stamp pads, and art paper were used during probes for generalization to novel art materials. To maintain Fran's interest in the materials, none of these art supplies were available at any time other than during research sessions (i.e., assessment and training sessions). Prior to every assessment and training session, Fran selected from an array of three options (e.g., Color Wonder paints, squeeze paints, and dot paints), which art supplies she wanted to use for that session. This daily preference assessment was important to prevent boredom or disliked materials from confounding Fran's motivation to talk during sessions. Researchers recorded Fran's choices to determine whether she repeatedly selected one type of art material. Fran independently varied her choice from day to day and session to session.

Development of scripts. Visual programmed stimuli (i.e., written scripts) were selected with the intent to make the natural antecedent stimuli (i.e., table, conversation partner, toys), which were visual, more salient in the pairing process. Although impaired significantly by limited social communication skills and restricted patterns of behavior, Fran had extensive experience with books and text. At the time of the study, she was able to recognize many familiar words and quickly learn new words. Early decoding ability is not unusual for children with autism (Newman, Macomber, Naples, Babitz, Volkmar, \& Grigorenko, 2007) and given many children with autism are also visual learners text may have several advantages over other forms of response prompts (Quill, 1997). 
Textual scripts were written to be applicable to all types of art materials used in the conduct of this study. Script sheets consisted of an $8 \frac{1}{2} \times 11$ inch cardstock on which five textual scripts (i.e., multiple exemplars) were typed in large print. For each language target (e.g., prepositions, coordinating conjunctions, subordinating conjunctions), a set of three script sheets were employed, each with a different order of the same five scripts. In other words, there were five scripts (textual line) for each of the three language targets (see Table 1), but they were printed in different orders creating three separate script sheets for each target. Of the five preposition scripts, two included the word on, two included the word with, and one included the word in. For coordinating conjunctions, three scripts included the word and, and two scripts included the word but. Three scripts included the subordinating conjunction word when and two scripts included the subordinating conjunction word because. Prepositions were introduced first and these scripts were created so that the later targets were not included in the scripts. However, some coordinating conjunction scripts contained prepositions, and some subordinating conjunction scripts contained prepositions and coordinating conjunctions. "I love to paint with this" is an example of a preposition script; "I love to play and paint with you" is an example of a coordinating conjunction script; "I love to paint with you because you're fun" and "It's messy when I get it on my hands and fingers" are examples of subordinating conjunction scripts. In these examples, the current targets are underlined and the previous targets are italicized. We created the scripts so that, once a new target was introduced, Fran continued to receive some exposure to previously learned language features. Also, the introduction of language targets into intervention roughly aligned with typical emergence of language features - typical children begin to use prepositions and coordinating conjunctions before using subordinating conjunctions (Justice \& Ezell, 2002; Vasilyeva, Waterfall, \& Huttenlocher, 2008). 
On the script sheets, to the left of each script (textual line) was a small picture of Fran $(1 / 2$ inch $x \frac{1}{2}$ inch), which we refer to as an icon. This icon marked each of the five scripts on the script sheet that Fran was to read aloud and served as a non-specific discriminative stimulus like the quotation marks in the Krantz \& McClannahan (1993) study. The icons were placed on the script sheets in case Fran was unable to produce the trained targets and/or statements in the absence of all programmed stimuli. The icons proved useful in facilitating the transfer of stimulus control for the third and most difficult language target, subordinating conjunctions. After five sessions in which Fran did not produce subordinating conjunctions in the assessment sessions, we adjusted the procedures by removing the scripts from the script sheets leaving only the five icons on the script sheet. A generic script sheet containing only the five icons was made available during the assessment sessions while the training sessions continued as planned using the full scripts.

Teaching the scripts. Between baseline and intervention phases, instructors taught Fran to read each script for the new language target in an instructional context (i.e., not in conversation while painting). Individual scripts were attached to $3 \times 5$ inch note cards and instructors used these to teach Fran to read each word of the text. All five scripts for each language target were taught simultaneously. After one or two days of frequent practice reading the scripts, they were introduced in training sessions (i.e., intervention phase began for that language feature). Because we planned to train loosely, we did not require Fran to read each script perfectly before beginning the transfer of stimulus control procedures in the conversation context (i.e., training sessions). This was important because we did not want her to memorize the scripts, which would have led to restricted stimulus control; however, if given sufficient time to practice Fran had the capability of reading each word accurately. After the scripts' introduction 
into daily training sessions, Fran continued to practice reading the scripts in an instructional context at least once a day. However, when a new language feature entered into intervention, the previous set of five scripts (e.g., preposition scripts) was no longer used in training sessions or for additional practice. Fran only practiced the new set of five scripts for the current language target (e.g., coordinating or subordinating conjunction scripts) on note cards and only the new set was presented on script sheets in training sessions. Even though the previous targets' scripts were discarded and never reappeared, the later targets' scripts included earlier targets (see examples above and in Table 1).

Assessment session procedures. Assessment sessions began when the conversation partner (i.e., instructor, mother, or peer) placed art supplies (in a rectangular container) on the table. At the same time, the partner started a digital voice recording device, which also tracked the length of the session. Fran and the conversation partner unpacked the materials and began painting. The conversation partner waited for Fran to begin the conversation. However, if Fran did not initiate the conversation within approximately 20 seconds of unpacking the materials, the conversation partner began talking about his/her own artwork. The conversation partner spoke naturally, modeled good talking, and replied to Fran's attempts to converse (natural contingencies of reinforcement). After three minutes, the conversation partner said, "It's time to clean up. Thanks for playing." Although textual scripts were never present during assessment sessions, for subordinating conjunctions we briefly introduced icons without textual scripts.

Training session procedures. Prior to training sessions, the first author selected one of the three script sheets out of a folder without regard to an order to create a semi-random rotation of script sheets. The first author and a research assistant served as prompters and stood behind Fran during training sessions. Training sessions began when the conversation partner (i.e., 
instructor) placed the materials and script sheet (in a rectangular container) on the table. Fran unpacked the script sheet with the materials and placed it to the side of the table. If necessary, the prompter provided hand over hand prompting to help Fran take out the script sheet and place it near her but out of the way of her painting. To facilitate spontaneity of Fran's language, the conversation partner waited for Fran to initiate the conversation. If Fran did not begin speaking within approximately 20 seconds of unpacking the art supplies, the prompter manually guided her hand toward the script sheet by holding Fran's forearm and letting go as soon as Fran began pointing to the script. This was sufficient to prompt Fran to read the script and initiate the conversation. Using both of her hands on the sides of Fran's head, the prompter gently oriented Fran's head toward the conversation partner as needed. Once conversation began, the prompter waited for approximately 10 seconds of no talking before executing manual guidance to help Fran point to the next script line. After the first few training sessions, only a few manual prompts were required per training session. Twice during the training phase for subordinating conjunctions the prompter provided a vocal prompt when Fran became frustrated because she was unable to read the script accurately. Otherwise, the prompter remained quiet during training sessions. Instructors spoke naturally, talking about their own artwork and replying to Fran's comments. When Fran had read all five scripts, the conversation partner said, "It's time to clean up. Thanks for playing." Training sessions lasted approximately five minutes; although the duration depended on how much Fran spoke in addition to the scripts.

Procedural fidelity. Prior to their involvement in the study, instructors and the research assistant who served as a prompter attended a training meeting consisting of a discussion of procedures, demonstration, and role-playing. Instructors were trained on five specific behaviors that were critical for being the conversation partner during assessment and training sessions: 1) 
place the art materials on the table without talking, 2) allow Fran to speak first or wait 20 seconds, 3) alternate speaking with Fran like in natural conversation, 4) respond to Fran's conversation statements enthusiastically but do not praise talking, and 5) when Fran is finished with her lines or three minutes have passed say, "Time to clean up. Thanks for playing." The prompters were trained on six steps: 1) stand behind Fran, 2) do not talk to Fran, 3) wait 20 seconds before prompting the start of the conversation, 4) after the conversation has begun, wait 10 seconds of no talking before prompting Fran to the next script, 5) use manual guidance holding Fran's forearm to help her point to the next script, and 6) if necessary, gently orient Fran's head toward the conversation partner. During the training of procedures, instructors and the prompter completed mock trials of assessment and training sessions while the first author recorded correct completion of steps using a fidelity checklist. The same fidelity checklist was used to examine the extent to which assessment and training procedures were implemented as intended. With respect to assessment procedures, $32 \%$ of the sessions across all phases and conversation partners were observed for fidelity and the average fidelity of implementation was $98 \%(80-100 \%)$. With respect to training sessions, which included the conversation partner and the prompter, $31 \%$ of the sessions were observed for fidelity with an average fidelity of implementation of $99 \%(93-100 \%)$.

Data analysis and agreement. Within two hours of completing the assessment session, the instructor listened to the digital recording and transcribed Fran's conversational exchanges word for word including grammatical errors and word deletions. When transcribing, the instructor stopped and replayed the recording or parts of the recording as many times as necessary. The instructor analyzed the transcript for number of language targets and calculated separate scores for prepositions, coordinating conjunctions, and subordinating conjunctions. To 
examine the consistency of scoring, the first author reviewed $36 \%$ of the transcripts across all phases including generalization probes and calculated independent scores for prepositions, coordinating conjunctions, and subordinating conjunctions. Based on the three language features, each transcript contained three opportunities for agreement or disagreement. Scoring agreement was calculated using the following formula: number of agreements divided by the number of agreements plus disagreements multiplied by $100 \%$. Interscorer agreement was $94 \%$ with three discrepancies for prepositions. Because these disagreements were with the word on, it is likely they were missed due to oversight and not because there was disagreement regarding how to classify the word.

Assessment transcripts were also examined to identify who initiated the conversation. During assessment sessions, the conversation partner waited 20 seconds to allow Fran time to begin the conversation. If she had not initiated the conversation within 20 seconds, the conversation partner started talking about his/her own artwork. Each assessment session was marked as Fran initiated or conversation partner initiated. Only seven sessions (four daily assessment sessions with the instructors and training materials and three generalization probes) constitute the baseline condition for this analysis and 43 sessions make up the training condition. Percent of conversations initiated by Fran was calculated for baseline and intervention phases.

Each of Fran's uses of the target language features were analyzed with reference to the scripts we trained. Previous researchers have analyzed unprompted responses; however, in our data all responses are considered unprompted because data were collected in the absence of prompts in a naturalistic context. Therefore, a more appropriate analysis involved the extent to which Fran varied her responses while still using the targeted language features (i.e., response generalization). To determine whether Fran used the target words in a generalized manner, we 
calculated the percent of targets emitted after teaching that were unique or untrained uses of the target language feature. We defined untrained uses as the inclusion of a target word in a phrase that shared no other words with the script phrases except articles and pronouns such as the, $a$, and $m y$. For example, if the script we trained read "The colors are pretty on the paper" and Fran said, "I got paint on my fingers," this was considered an untrained use of the preposition on. The percent of untrained uses of targets was calculated for each of the three language features.

\section{Results}

The frequency of target words emitted during 3-minute assessment sessions for prepositions, coordinating conjunctions, and subordinating conjunctions is presented in Figure 1. During baseline sessions, Fran rarely used target language features during conversation about art. Once training began with prepositions, the frequency of use increased in a variably ascending pattern with a range of zero to four. Baseline levels of coordinating and subordinating conjunctions remained low when only prepositions were targeted during script and generalization training. When coordinating conjunctions were trained, Fran increasingly used coordinating conjunctions with a range from zero to four and continued to use prepositions. Coordinating conjunctions followed a variable pattern with clear level changes. Subordinating conjunctions did not improve immediately following the introduction of training. After five days of training, Fran had not emitted a subordinating conjunction in an assessment session. We briefly introduced a script sheet into assessment sessions; however, scripts were removed so only the icons (small pictures of Fran; non-specific discriminative stimuli) were displayed on the script sheet. This transfer of stimulus control strategy was sufficient to get Fran to use the subordinating conjunctions during assessment sessions and we withdrew the script sheet from assessment sessions after eight sessions. In the five generalization probes following the removal 
of the script sheet with icons, Fran continued to use subordinating conjunctions when the script sheet was not present with a range from zero to four.

$<$ Fig. 1 here $>$

During baseline, Fran initiated conversation twice in the seven assessment sessions (29\%), whereas during the intervention phase Fran began the conversation in $86 \%$ of the sessions. Calculated percentages of untrained variations of the targeted language features were $81 \%$ for prepositions, $78 \%$ for coordinating conjunctions, and $16 \%$ for subordinating conjunctions.

\section{Discussion}

The purpose of this study was to explore the feasibility of combining several generalization-enhancing strategies and script training procedures to affect the generalized use of complex language features of a child with autism. Results indicate that the integration of scripts and transfer of stimulus control procedures produced immediate improvements in prepositions and coordinating conjunctions and with the brief addition of non-specific discriminative stimuli the effect was achieved for subordinating conjunctions. There are several reasons these results are significant. First, data were recorded during assessment sessions only; rather than assessing performance during training. Because textual scripts were never present during assessment sessions and they reflected a natural context for talking, we essentially tested stimulus generalization every day. This intermittent exposure to the same conditions without the scripts could have served as a variation of script fading like in Woods and Poulson's (2006) study. In addition to our continuous measure of stimulus generalization, we also conducted situation generalization probes with typical peers and Fran's mother, in Fran's home and in her kindergarten classroom, and with art materials that were not used during training. After training, 
Fran used the targeted language features substantially more when probed for generalization than during baseline and the improvements maintained after 4-weeks of no training. Second, assessment sessions occurred daily before intervention sessions so the test for transfer to a nontraining context occurred 24 hours after training. Improvements detected following a significant delay from teaching are more robust than those that are recorded during training sessions. This is an important departure from most of the script training literature. Third, improvements in the use of prepositions and coordinating conjunctions were noticed after only one generalization training session. In previous script training with fading research, several training sessions were necessary before equivalent improvements occurred.

In general, the degree of change observed may not appear to be substantial because the frequency of targets increased, at most, by four and results were variable. However, it is important to note that while we programmed five opportunities for the target language feature to occur during training sessions, opportunities for Fran to use prepositions, coordinating conjunctions, and subordinating conjunctions during assessment sessions were not programmed. Considering that Fran was free to emit any of the language targets and it took approximately 5 minutes for Fran to read all five of the scripts during training sessions, it would be unnatural for Fran to use any one of these target features more than 3-5 times during a 3-minute conversation. Because we used frequency of targets rather than percent of opportunities, we inspected the transcripts post hoc to determine how often Fran attempted to use a trained phrase but omitted the targeted language feature, constituting a failed opportunity. Interestingly, Fran seldom attempted a phrase in which the target features were unsuccessfully used. During baseline, Fran's language productions were simple phrases such as "You paint this one," "Purples gonna change," and "I make good grass." Instead of saying, "The butterfly is orange and blue" Fran 
said, "The butterfly is orange. The butterfly is blue," avoiding the need to use a coordinating conjunction. In contrast, Fran said, "My flower is yellow and red" following training on coordinating conjunctions. In general her language productions became more complex after training, suggesting a meaningful language improvement. For instance during the follow-up session, Fran said, "You can paint a sky on your paper" and "I want to use that color when you're done."

As an estimate of spontaneity, we calculated the percent of assessment sessions in which Fran started the conversation. Procedures allowed for a 20 -second window from the time art materials were available to when the conversation partner started talking. If Fran started talking within this window, we considered the conversation initiated by her. In baseline, Fran began the conversation in less than a third of the sessions. She typically unpacked the art materials and started painting without acknowledging her conversation partner or attempting to gain his or her attention. Once training began, Fran was much more likely to begin the conversation; often she started talking about the paints and telling her conversation partner what she was going to create within a few seconds of the paints' availability. The only methodological aspects of this study designed to increase conversation initiations were the instructor's delayed initiations during assessment sessions and prompting during training sessions for Fran to use the scripts to begin the conversation. Even though it is possible that these components of the training procedures contributed to increased initiations, the current study does not allow for definitive conclusions regarding an experimental effect.

Response generalization was assessed by calculating the percent of untrained uses of the targeted language features. We intended to teach prepositions, coordinating conjunctions, and subordinating conjunctions as response classes using multiple exemplars (i.e., multiple targets 
within each class and multiple scripts for each target) and loose training of the scripts themselves (i.e., Fran was not required to be a proficient script reader before extending training to the conversation context). Because we introduced all five scripts at once, it slowed down her rate of decoding acquisition and prevented restricted stimulus control from developing. The effect of these strategies was evident when Fran struggled to remember the trained script word for word during assessment sessions. On occasion, Fran said, speaking to herself, "That's not it. Try again." After a few attempts to say it as she learned it she abandoned the attempt and changed it to suit the current situation, which we anticipated. Over time, the untrained uses increased for all three language targets so in the final assessment sessions, few of the targets emitted were contained in trained phrases. The percent of untrained uses was greatest for prepositions and lowest for subordinating conjunctions. This pattern was not unexpected. The procedures allowed for substantially more exposures to scripts and a wider variety of scripts with prepositions than subordinating conjunctions. Also, it is likely that due to the amount of time elapsed since direct training on prepositions versus coordinating conjunctions versus subordinating conjunctions. Lastly, the percent of untrained responses followed the developmental sequence of the three language targets selected (Justice \& Ezell, 2002; Vasilyeva et al., 2008). Prepositions and coordinating conjunctions were possibly emerging in her language repertoire, whereas subordinating conjunctions were not. Occasional baseline uses of prepositions and coordinating conjunctions support this notion. It is noteworthy that for Fran to produce a subordinating conjunction, the length of the sentence had to be longer than what she had ever produced in conversation.

One interesting finding of this study involves the temporary inclusion of the non-specific discriminative stimuli (i.e., icons) in the assessment sessions. In Krantz and McClannahan 
(1993), the quotation marks, which they were unable to eliminate, evoked a variety of conversation statements. While this was a limitation in their study because it was unanticipated, the intentional use of non-specific discriminative stimuli to promote spontaneity and generalization of language may have positive effects. We constructed our script sheets to include the visual icons in the pairing process. When transfer of language to assessment sessions did not occur for subordinating conjunctions, we elected to add the least interfering prompts possible and evaluate their effect before introducing the more intrusive textual scripts. The effect of this minimal prompting tactic was immediate. We believe that the icons enhanced the saliency of the paired training and generalization stimuli and facilitated the transfer of stimulus control. Considering there is not one to one correspondence between a non-specific discriminative stimulus and a specific response form, this strategy, if arranged thoughtfully, can help avoid restrictive stimulus control common during acquisition training. While this conclusion is only speculative, the effect of this type of strategy is worth exploring in future research.

Despite the importance of the findings, this study is limited in a number of ways. The most significant is that we investigated the effects of the combination of script training and transfer of stimulus control procedures with only one participant. Even though a multiple baseline across behaviors was employed, notable effects were observed for only two of the three targets. Additional tactics were necessary for improvements to be shown with subordinating conjunctions. Thus, little can be concluded with respect to experimental effect of the intervention. Further replication with additional participants is needed.

Our procedures for training loosely (i.e., no specific performance criterion before beginning generalization training) and using natural contingencies of reinforcement (i.e., we did 
not prescribe what conversation partners should and should not say) limit the replicability of our study. Even so, results of pilot research suggested that those aspects were worth compromising. For example, in pilot research, we found that using a performance criterion for script reading inadvertently facilitated tighter stimulus control than we desired. The repeated association of the programmed discriminative stimuli and trained responses during acquisition impaired the development of response variations and language use was difficult to maintain when the scripts were absent. In addition, we restricted what the conversation partners could say, which led to a very unnatural conversation exchange that did not function as reinforcement for our participants. The children quickly discriminated between natural and "training" conversation, which likely impeded generalization. To create a more natural and reinforcing conversation context in the current study, we did not restrict what the conversation partners could say, but this adds another limitation. It is possible that Fran's language productions were related in some way to the conversation partner's use of the target language features. Though no obvious connection was discovered through a review of the transcripts, this potential confound was not controlled for experimentally. We suggest further researchers examine methods for maximizing natural consequences and loose training while balancing the need for prescribed research procedures.

\section{Summary and Conclusions}

Developing a functional conversation repertoire requires acquisition of language and further, it requires that newly-acquired language expressions be used with some spontaneity; that is, that they be under the control of stimuli in the child's natural environment. Although these are necessary attributes, they are not sufficient. It is also crucial that the language be used in the variety of circumstances where it would be appropriate, even in circumstances different from those in which the training took place and with content different from that used in training. 
Moreover, language responses that are varied and flexible are more useful than those that are direct repeats of trained responses. Special attention to functional aspects of language such as spontaneity, stimulus/situation generalization, and response flexibility is necessary for teaching useful language to children with autism.

The exploratory nature of this study suggests further examination of the phenomena at play is warranted. It should be noted that because there were so many generalization-enhancing tactics employed in this case study it is near impossible to speculate regarding the role each had. An important next step could be to evaluate the impact of each generalization-enhancing procedure independently, in a comparative fashion. It might be that they are more powerful in combination; however, that is currently unknown. Nonetheless, the current study was systematic in its logic for combing procedures and therefore, may serve as a model to practitioners when designing interventions for children with autism for maximum generalization. Moreover, it highlights areas where the literature is sparse. There are too few studies in which the strategies for transferring stimulus control are investigated as the primary independent variables. The majority of intervention research with children with autism addresses acquisition of behaviors and the main generalization method employed is train and hope (Stokes \& Baer, 1977). The application of social communication interventions has certainly advanced beyond train and hope, yet such technology is rarely featured prominently in research.

In this study we translated generalization technology into practical and implementable tactics. First, we employed natural contingencies of reinforcement by allowing Fran to choose the activity for each session and ensuring the conversation partner spoke naturally and enthusiastically in response to Fran's conversation statements. In our pilot study, participants may or may not have liked the toys we used and in some cases edible reinforcers were used. We 
expect that practitioners experience the greatest obstacles to training for generalization for children who are not easily motivated by social reinforcers. However, if natural contingencies of reinforcement are essential to promote spontaneity and generalization of language, then improving the capacity of the natural consequences to function as reinforcers should be a priority. Our second tactic was to use multiple exemplars. Although multiple exemplars were employed in the pilot study, they were introduced sequentially. The simultaneous introduction of several scripts containing the target language feature and the inclusion of previously learned targets in later scripts likely contributed to the response variation observed in prepositions and coordinating conjunctions. Fran was exposed to many different ways to use each target, which potentially contributed to her response flexibility. Training loosely was our third tactic, which can be seen in several procedures. The most obvious is that we did not require a reading performance criterion before introducing the generalization strategies. Other strategies though, were the intentional loose conversation allowed from the partners, the daily exposure to the nontraining conditions of the assessment sessions, and exposure to several scripts at once reducing Fran's accuracy of script reading. Finally, we programmed for common stimuli in both treatment and assessment sessions. These stimuli were taken directly from a context we anticipated would be natural for Fran in the near future (e.g., table, art materials, conversation partner). Although we would have preferred to train using typical peers because they are more natural conversation partners than instructors, they were unavailable in her preschool program. And instead of programming using them, we were able to test whether our other strategies were sufficient to produce generalization to non-training conversation partners.

Although these strategies were successful in Fran's situation, we strongly encourage additional research to verify these effects using stronger controls and more participants. These 
findings and the logical conceptualization of relevant independent variables may assist researchers and practitioners in effecting significant generalized behavior change. Nonetheless, a logical analysis should not supplant an experimental analysis. 


\section{References}

American Psychiatric Association. (2000). Diagnostic and statistical manual of mental disorders (4th ed., text revision). Washington, DC: Author.

Argott, P., Townsend, D. B., Sturmey, P., \& Poulson, C. L. (2008). Increasing the use of empathic statements in the presence of a non-verbal affective stimulus in adolescents with autism. Research in Autism Spectrum Disorders, 2, 341-352.

Brown, J. L., Krantz, P. J., McClannahan, L. E., Poulson, C. L. (2008). Using script fading to promote natural environment stimulus control of verbal interactions among youths with autism. Research in Autism Spectrum Disorders, 2, 480-497.

Carr, E. G. (1982). Sign language. In R. L. Koegel, A. Rincover, \& A. L. Egel (Eds.), Educating and understanding autistic children (pp. 142-157). San Diego: College-Hill Press.

Carr, E. G., \& Kologinsky, D. (1983). Acquisition of sign language by autistic children. II: Spontaneity and generalization effects. Journal of Applied Behavior Analysis, 16, $297-$ 314.

Carter, M., \& Hotchkis, G. D. (2002). A conceptual analysis of communicative spontaneity. Journal of Intellectual and Developmental Disabilities, 27, 168-190.

Charlop, M. H., Schreibman, L., \& Thibodeau, M. G. (1985). Increasing spontaneous verbal responding in autistic children using a time delay procedure. Journal of Applied Behavior Analysis. 18, 155-166.

Chiang, H., \& Carter, M. (2008). Spontaneity of communication in individuals with autism. Journal of Autism and Developmental Disorders, 38, 693-705.

Cooper, J. O., Heron, T. E., \& Heward, W. L. (Ed.). (2007). Applied behavior analysis, $2^{\text {nd }}$ Ed. Upper Saddle River, NJ: Pearson Education, Inc.

Halle, J. W. (1987). Teaching language in the natural environment: An analysis of spontaneity. 
Journal of the Association for Persons with Severe Handicaps, 12, 28-37.

Justice, L.M., \& Ezell, H.K. (2002). The syntax handbook: Everything you learned about syntax (but forgot). Eau Claire, WI: Thinking Publications.

Krantz, P. J., \& McClannahan, L. E. (1993). Teaching children with Autism to initiate to peers: Effects of a script-fading procedure. Journal of Applied Behavior Analysis. 26, 121-132.

Koegel, L. K. (2000). Interventions to facilitate communication in autism. Journal of Autism and Developmental Disorders, 30, 383-391.

MacDuff, J. L., Ledo, R., McClannahan, L. E., \& Krantz, P. J. (2007). Using scripts and scriptfading procedures to promote bids for joint attention by young children with autism. Research in Autism Spectrum Disorders, 1, 281-290.

McClannahan, L. E., \& Krantz, P. J. (2005). Teaching conversation to children with autism: Scripts and script fading. Bethesda: Woodbine House.

McConnell, S. R. (2002). Interventions to facilitate social interaction for young children with autism: Review of available research and recommendations for educational intervention and future research. Journal of Autism and Developmental Disorders, 32, 351-372.

Newman, T. M., Macomber, D., Naples, A. J., Babitz, T., Vokmar, F., \& Grigorenko, E. L. (2007). Hyperlexia in children with autism spectrum disorders. Journal of Autism and Developmental Disorders, 37, 760-774.

Quill, K. (1997). Instructional considerations for young children with autism: The rationale for visually cued instruction. Journal of Autism and Developmental Disorders, 27, 697-714.

Reagon, K. A., \& Higbee, T. S. (2009). Parent-implemented script-fading to promote playbased verbal initiations in children with autism. Journal of Applied Behavior Analysis, 
$42,659-664$.

Robertson, S. A. (2007). Assessment of preschool and early school-age children with developmental language disorders. In A. G. Kamhi, J. J. Masterson, \& K. Apel (Eds.), Clinical decision making in developmental language disorders, (pp. 39-54). Baltimore, MD: Paul H. Brooks.

Sigafoos, J., \& Reichle, J. (1993). Establishing spontaneous verbal behavior. In R. A. Gable \& S. F. Warren (Eds.), Strategies for teaching students with mild to severe mental retardation. Baltimore, MD: Paul H. Brookes.

Stevenson, C. L., Krantz, P. J., \& McClannahan, L. E. (2000). Social interaction skills for children with autism: A script-fading procedure for nonreaders. Behavioral Interventions, $15,1-20$.

Stokes, T. F., \& Baer, D. M (1977). An implicit technology of generalization. Journal of Applied Behavior Analysis, 10, 349-367.

Woods, J., \& Poulson, C. L. (2006). The use of scripts to increase the verbal initiations of children with developmental disabilities to typically developing peers. Education and Treatment of Children. 29, 437-457.

Vasilyeva, M., Waterfall, H., \& Huttenlocher, J. (2008). Emergence of syntax: Commonalities and differences across children. Developmental Science, 11(1), 84-97. 
Table 1

List of Scripts for Each Language Target

\begin{tabular}{ll}
\hline Language Targets & \multicolumn{1}{c}{ Scripts } \\
\hline & I love to paint with this. \\
Do you like to paint with me? & I'm going to pain in this spot. \\
The colors are pretty on the paper. & It goes on the paper. \\
& I love to play and paint with you. \\
Painting is fun, but messy. \\
Coordinating & I like painting and making beautiful pictures. \\
& I paint on my paper but you paint on yours. \\
& Your picture is pretty and colorful. \\
Subordinating & I love to paint with you because you're fun. \\
Conjunctions & Painting is fun, but messy when it gets on me. \\
& It's messy when I get it on my hands and fingers. \\
& I like my picture because it's beautiful. \\
& Can I have that color when you're done? \\
\hline
\end{tabular}




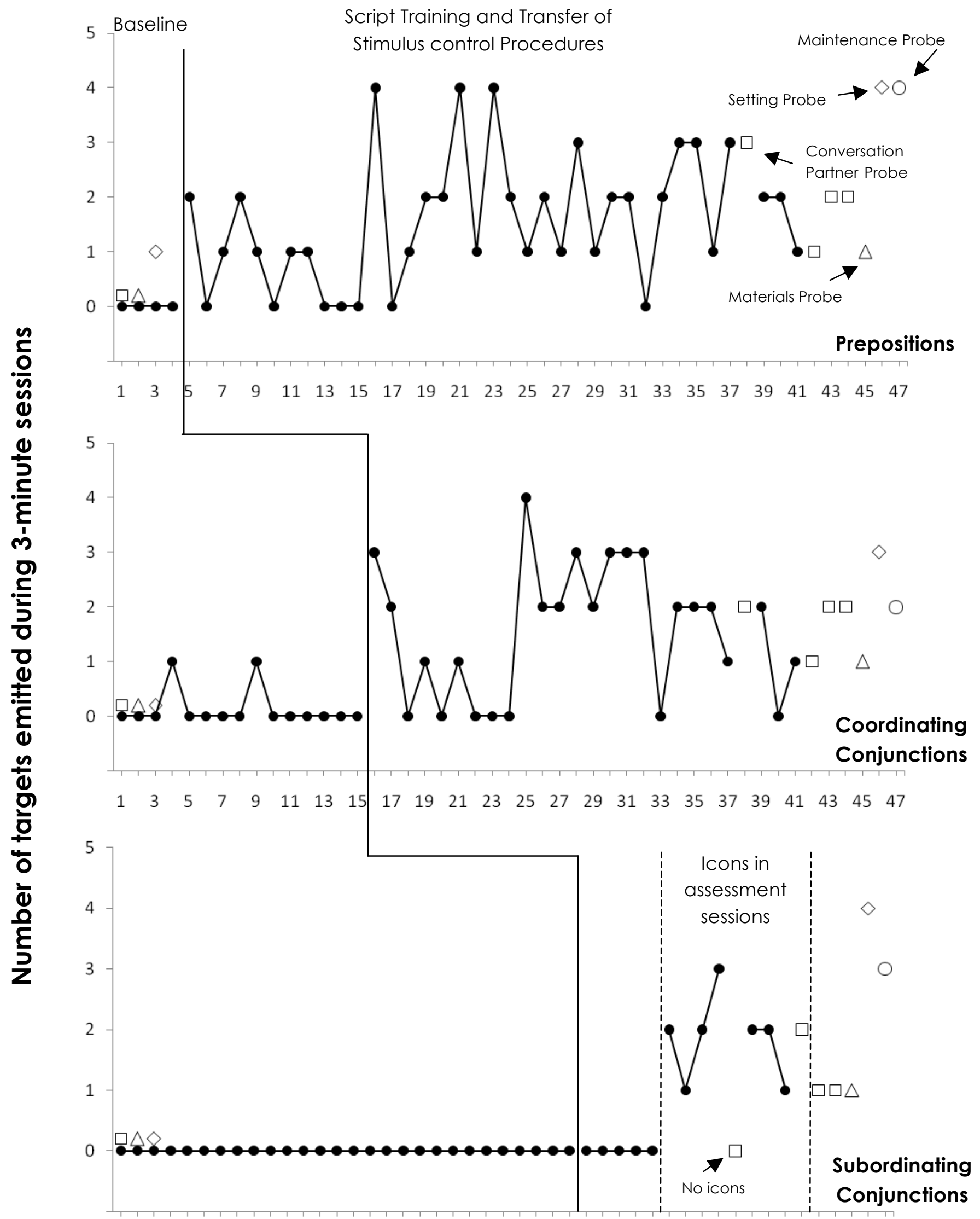

$\begin{array}{llllllllllllllllllllllll}1 & 3 & 5 & 7 & 9 & 11 & 13 & 15 & 17 & 19 & 21 & 23 & 25 & 27 & 29 & 31 & 33 & 35 & 37 & 39 & 41 & 43 & 45 & 47\end{array}$

\section{Sessions}


Figure 1. Number of prepositions, coordinating conjunctions, and subordinating conjunctions emitted during 3-minute daily assessment sessions. 